Cita: Fin, G.; Jesus, J. A.; Benetti, M.; Nodari Júnior, R. J. (2022). La práctica de actividad física en mujeres con cáncer de mama: asociación entre factores motivacionales y características dermatoglíficas. Cuadernos de Psicología del Deporte, 22(1), 176-189

\title{
La práctica de actividad física en mujeres con cáncer de mama: asociación entre factores motivacionales y características dermatoglíficas
}

\section{The practice of physical activity in women with breast cancer: association between motivational factors and dermatoglyphic characteristics}

\section{A prática da atividade física em mulheres com câncer de mama: associação entre fatores motivacionais e características dermatoglíficas}

\author{
Fin, Gracielle ${ }^{1}$, Jesus, Josiane Aparecida de ${ }^{1}$, Benetti, Magnus², Nodari Júnior, Rudy José ${ }^{3}$ \\ ${ }^{1}$ Universidade do Oeste de Santa Catarina, Mestrado em Biociências e Saúde, Joaçaba, \\ Brasil, ${ }^{2}$ Universidade do Estado de Santa Catarina, Centro de Ciências da Saúde e do Esporte, \\ Florianópolis, Brasil; ${ }^{3}$ Salus Dermatoglifia, Sientific Research Department, Joaçaba, Brasil
}

\begin{abstract}
RESUMO
O objetivo foi investigar a associação dos construtos da teoria da autodeterminação e características dermatoglíficas no comportamento para a prática de atividade física em mulheres com câncer de mama. Participaram 104 mulheres que responderam às escalas de prática de atividade física, percepção de competência, autonomia para a prática de atividade física, satisfação com a vida e características dermatoglíficas. No grupo de mulheres que praticava atividade física antes do diagnóstico e continuou a prática durante o tratamento, a percepção de competência, regulação autônoma, satisfação com a vida e nível de atividade física foram maiores. As variáveis dermatoglíficas mostraram que, no polegar e indicador da mão direita, a distribuição dos verticilos em $\mathrm{S}$ foi maior nas mulheres com regulação controlada para a prática de atividade física. A percepção de competência e a regulação autônoma são importantes para a manutenção da prática de atividade física durante o tratamento do câncer de mama.
\end{abstract}

Palavras-chave: Teoria da autodeterminação; Exercício físico; Competência; Autonomia; Câncer.

\section{RESUMEN}

El objetivo fue investigar la asociación de los constructos de la teoría de autodeterminación y las características dermatoglíficas en el comportamiento para la práctica de actividad física en mujeres con cáncer de mama. Participaran 104 mujeres que contestaron las escalas de práctica de actividad física, percepción de competencia, autonomía para la práctica de actividad física, satisfacción con la vida y dermatoglifia. En el grupo que practicaba actividad física antes del diagnóstico y continuaron durante el tratamiento, la percepción de competencia, regulación autónoma, satisfacción con la vida y nivel de actividad física eran mayores. En la dermatoglifia, en el pulgar y índice de la mano derecha, la distribución de verticilos en $\mathrm{S}$ fue mayor en las mujeres con regulación controlada para la práctica de actividad física. La percepción de competencia y la regulación autónoma son importantes para mantener la práctica de actividad física durante el tratamiento del cáncer de mama.

Palabras clave: Teoría de la autodeterminación; Ejercicio físico; Competencia; Autonomía; Cáncer. 


\title{
Actividad física, motivación y dermatoglífia en mujeres con cáncer de mama
}

\begin{abstract}
The aim was to investigate the association of constructs from the theory of self-determination and dermatoglyphic characteristics on the behaviour to the practice of physical activity in women with breast cancer. 104 women answered questions about the practice of physical activity, perception of competence, autonomy to the practice of physical activity, satisfaction with life, and dermatoglyphics. In the group that practiced physical activity before diagnosis and continued during treatment, the perception of competence, autonomous regulation, life satisfaction and level of physical activity were higher. To dermatoglyphics, the thumb and index finger of the right hand, the distribution of whorls in S pattern was higher in women with controlled regulation for physical activity, more studies are necessary to verify how these variables behave in the scope of breast cancer. The perception of competence and the autonomous regulation are important for the maintenance of the practice of physical activity during the treatment. Keywords: Self-Determination Theory; Physical exercise; Competence; Autonomy; Cancer.
\end{abstract}

\section{INTRODUÇÃO}

A inatividade física aumenta o risco de câncer de mama na pós-menopausa e possivelmente na prémenopausa, embora sejam propostos diferentes mecanismos biológicos (Neilson, Farris, Stone, Vaska, Brenner \& Friedenreich, 2017). Estudos comprovam que a atividade física está associada a um menor risco para vários tipos de câncer, mas apenas o câncer de cólon e de mama apresentam associações fortes ou evidências altamente sugestivas (de Rezende et al., 2018). Mesmo assim, a prática de atividade física ainda é um fator desafiador para as pessoas que se encontram em tratamento oncológico e para os profissionais da saúde. Muitos têm receio e podem não reconhecer inicialmente os benefícios deste comportamento, uma vez que o tratamento para o câncer pode gerar alguns desconfortos específicos que interferem na realização de atividades físicas, como fadiga severa, anemia, cateteres permanentes, disfunções neurológicas, entre outros (Rock et al., 2012).

A adesão para a prática de atividades físicas durante o tratamento pode também sofrer influência do encaminhamento médico/clínico direcionado (Livingston et al., 2015). Outros fatores como fadiga, dor, falta de motivação e falta de disciplina podem influenciar a adesão ou permanência na atividade física durante o tratamento do câncer, sendo que em pesquisa recente observou-se que $75 \%$ das pessoas em tratamento para o câncer reduzem os níveis de atividade física após o diagnóstico (Romero, Li \& Mao, 2017), mesmo com estudos sugerindo que a atividade física é bem tolerada durante e após o tratamento sem eventos adversos (Speck, Courneya, Mâsse, Duval, \& Schmitz, 2010).
Pesquisadores vêm apoiando seus estudos sobre o comportamento relacionado a saúde na Teoria da Autodeterminação (TAD), encontrando resultados positivos nos comportamentos e, indiretamente, melhora na saúde física e psicológica (Ntoumanis et al., 2020). A TAD (Deci \& Ryan, 2017) vem sendo utilizada de maneira substancial no contexto da saúde nos últimos 15 anos (Chatzisarantis, Hagger, Kamarova \& Kawabata, 2012; Duda et al., 2014; Halvari, Healey, Olafsen, Byrkjeland, Deci \& Williams, 2017; Husted, Thorsteinsson, Esbensen, Gluud, Winkel, Hommel \& Zoffmann, 2014; Ntoumanis et al., 2020; Zhou, 2021). Trata-se de uma teoria desenvolvida e apresentada por Deci e Ryan, (2017) como um modelo de explicação da motivação humana centrada na personalidade e nos contextos sociais, que se diferenciam em motivação autônoma ou controlada. Esta perspectiva tem o objetivo de explicar a motivação e o comportamento humano, baseada em diferentes orientações motivacionais, influências do contexto e percepções interpessoais (Deci \& Ryan, 2000).

Para a TAD o comportamento humano é motivado por três necessidades psicológicas básicas e universais: autonomia, competência e relacionamento. Sendo que a autonomia se refere aos esforços da pessoa em ser o agente das suas ações, agindo como o responsável em atingir seus objetivos, determinando seus próprios comportamentos. A competência está diretamente relacionada com o sentimento de eficácia, diante de alguma ação, que faz com que as pessoas se sintam seguras em suas possibilidades e realizem novas atividades para aumentar sua competência. Por sua vez, a necessidade de relacionamento ou vínculo, se refere ao esforço em criar relações, e preocupar-se com os outros, sentindo satisfação com suas relações 


\section{Fin et al.}

sociais, sendo considerada em duas dimensões, a de sentir-se aceito e íntimo com os outros (Deci \& Ryan, 1991). A satisfação das necessidades psicológicas básicas está associada ao prazer, ao esforço na realização de atividades e a outros resultados positivos (Deci \& Ryan, 2017), e quando frustradas resultam em consequências negativas no bem-estar e saúde pessoal (Ryan \& Deci, 2000).

No domínio da saúde estudos baseados na TAD examinam as relações entre os constructos da teoria e os comportamentos de saúde (por exemplo, atividade física, abstinência do tabagismo) e os índices de saúde (por exemplo, higiene dental, depressão, qualidade de vida, satisfação com a vida), com resultados que apontam a importância da percepção de competência e da regulação autônoma sobre a motivação (Oumrait et al., 2020; Ng, Ntoumanis, Thøgersen-Ntoumani, Deci \& Williams, 2012; Gillison, Rouse, Standage, Sebire \& Ryanet, 2019; Ntoumanis et al., 2020).

Além dos aspectos psicossociais que podem influenciar a adoção de comportamentos de saúde, há ainda características inatas que podem ser fatores determinantes sobre atitudes relacionadas à saúde. Os aspectos inatos de comportamento vêm sendo estudados e associados ao fenótipo em que os indivíduos são inseridos, como uma possibilidade de elucidar aspectos relacionados à influência do genótipo no desenvolvimento da personalidade adulta (Bouchard \& McGue, 1990), sendo encontrados estudos sugerindo a utilização da dermatoglifia como possível método de pesquisa científica também nas ciências psicológicas (Akbarova, 2018).

A dermatoglifia estuda as impressões digitais como indicadores de condições existentes várias semanas antes do nascimento de um indivíduo. Os padrões dermatoglíficos (desenhos), formados durante o período embrionário, são classificados por diferentes autores, sendo principalmente divididos em três ou quatro tipos: Arco (A) é o desenho formado por linhas basilares e marginais sem construção de deltas ou núcleos; Presilha Ulnar (LU) é o desenho formado por linhas basilares, marginais e nucleares de forma que permita a construção de apenas um delta e este, em relação ao núcleo da figura, está disposto no lado ulnar da mão; Presilha Radial (LR) é o desenho formado por linhas basilares, marginais e nucleares de forma que permita a construção de apenas um delta e este, em relação ao núcleo da figura, está disposto no lado radial da mão; Verticilo (W) ou Verticilo (S) é o desenho formado por linhas basilares, marginais e nucleares de forma que permita a construção de dois deltas e dois núcleos (Abramova, Nikitina, Izaak \& Kochetkova, 2000; Nodari Júnior \& Fin, 2016)

A Dermatoglifia, vem se mostrando uma possibilidade coadjuvante nas avaliações em saúde (Nodari Júnior \& Fin, 2016), uma vez que a individualidade biológica é um dos fatores observáveis quanto ao surgimento do câncer e pode ser também um fator que caracteriza certos tipos de comportamento. A literatura já demostra que as características dermatoglíficas podem servir como uma marca potencialmente útil e uma ferramenta de diagnóstico, juntamente com os métodos usuais, para identificar um grupo específico de indivíduos com predisposição para determinados tipos de câncer como o de mama (Bierman, Faith \& Stewart, 1988; Khandelwal, Mittal, Saijanani, Tuteja, Bansal, Bhatnagar \& Saxena, 2007; Sariri, Kashanian, Vahdat \& Yari, 2012; Yaneva et al., 2018). Também há estudos que correlacionam a dermatoglifia com a diabetes na meia-idade (Kahn, Graff, Stein \& Lumeyet, 2009) e outras doenças relacionadas ao estilo de vida (Kaur, 2019).

As características de personalidade e temperamento também vêm sendo estudadas considerando fatores biológicos e de herdabilidade (Ito \& Guzzo 2002), com estudos que indicam correlação entre as características dermatoglíficas e níveis de inteligência emocional (Kumari, Babu \& Kumar, 2014; Suresh \& Padmalatha, 2019). No contexto comportamental, a dermatoglifia surge como possível área de estudo. Em alguns casos na psicologia, o uso das marcas de individualidade biológica, como as impressões digitais, vem sendo estudadas considerando diferentes características psicológicas (Akbarova, 2018) e traços da personalidade (Serebrennikova, Gunas, Klimas, Ocheretna \& Shayuk, 2019)

Existem na literatura estudos mostrando a importância da autoeficácia e do prazer, como aspectos cognitivo e afetivo relacionados à prática de atividade física em pessoas com câncer (Leclair, Lebel \& Westmaas, 2021; Ungar, Wiskemann \& Sieverding, 2016), e outros estudos recentes que apresentam a relação entre os constructos da teoria da autodeterminação e o comportamento relacionado à saúde (Oumrait et al., 2020; Fenton et al., 2020; Kim, Chu, Oh, Shin, Jeon \& Lee, 2020). Todavia, não foram encontrados estudos 


\section{Actividad física, motivación y dermatoglífia en mujeres con cáncer de mama}

que identificam estas relações com pessoas em tratamento para o câncer de mama, bem como a relação com as caraterísticas inatas das impressões digitais, por meio da dermatoglifia.

Explora-se diferentes contextos e estratégias para se obter resultados ligados à saúde, neste sentido, o conhecimento dos aspectos comportamentais $\mathrm{e}$ biológicos relacionados ao estilo de vida do paciente oncológico contribui para a qualificação do serviço de saúde, por meio da implementação de protocolos de assistência para pessoas com câncer, impactando positivamente na saúde deste e em todo o ciclo social em que ele está inserido. Considera-se a ausência de estudos semelhantes anteriores e a hipótese de que mulheres com maior nível de satisfação dos constructos da teoria da autodeterminação apresentam também maior nível de atividade física, mesmo durante o tratamento para o câncer de mama, assim como pode existir a possibilidade de associação da dermatoglifia, considerando os aspectos inatos do comportamento. Assim, o objetivo deste estudo foi investigar a associação dos constructos da teoria da autodeterminação e das características dermatoglíficas com o comportamento relacionado à prática de atividade física em mulheres que se encontram em tratamento para o câncer de mama, considerando seu histórico de atividade física.

\section{MATERIAL E MÉTODOS}

\section{Participantes}

Participaram deste estudo 104 mulheres, com idades entre 25 e 81 anos $(M=54, D P=11)$ que apresentam diagnóstico de câncer de mama e realizam seu tratamento no setor de oncologia de um hospital da região meio oeste de Santa Catarina, Brasil.

\section{Instrumentos}

Características sociodemográficas e de estilo de vida. As participantes responderam a um questionário com informações referentes a características sociodemográficas (idade, data e local da avaliação, estado civil, escolaridade, emprego e renda) e de estilo de vida antes da doença (se praticava atividade física antes da doença, tempo de prática, quantos dias na semana e qual atividade)

Nível de Atividade Física. Foi utilizado o Questionário de Nível de Atividade Física Habitual (Habitual
Physical Activity Questionnaire) (HPAQ) (Baecke, Burema \& Frijters, 1982; Florindo \& Latorre, 2003), que é uma escala de autopreenchimento, constituída por 16 itens, que procura avaliar a atividade física habitual em três dimensões: atividade física no trabalho (AFT), exercício físico no tempo de lazer (EFL) e atividade física no tempo de lazer e locomoção (ALL), reportando-se aos últimos 12 meses. Os três domínios podem ser avaliados separadamente, sendo assim, neste estudo foram incluídas somente as dimensões de EFL e ALL. A razão de não incluir a $\mathrm{AFT}$ se deve em função da amostra deste estudo ser constituída por mulheres em tratamento para câncer de mama, estando, em grande parte, afastadas de suas atividades laborais. Também porque este trabalho se insere num estudo mais amplo sobre motivação e comportamentos de saúde, pretendemos um questionário curto, que permita diferenciar as participantes e, simultaneamente identificar comportamentos passíveis de serem alterados no sentido de promover a atividade física. $\mathrm{O}$ questionário completo poderia aumentar o número de itens, sem necessidade, tornando o questionário final longo. A avaliação dos exercícios físicos no lazer (EFL) é investigada mediante prática dos exercícios físicos regulares envolvendo modalidades específicas, divididas em três níveis de intensidade, de acordo com o gasto energético: leve, moderada e vigorosa. Sugerese que, para essa classificação, seja utilizado o compêndio de atividades físicas citado anteriormente (Ainsworth et al., 2000). Na avaliação das atividades de lazer e locomoção (ALL), as questões referem-se às atividades sedentárias (como assistir televisão), caminhar, andar de bicicleta e uma última questão sobre os minutos por dia em atividades de locomoção (como ir ao mercado ou andar de bicicleta). Para a determinação do escore total de AFH (ET) somam-se os escores AFT, EFL e ALL, neste estudo o escore final foi a soma de EFL e ALL.

Percepção de Competência. Foi utilizada a Perceived Competence Scale (PCS) (Williams \& Deci, 1996), traduzida para o português por Mestre e Pais Ribeiro (2008). A Escala é utilizada para avaliar o grau de competência percebida pelo sujeito na sua capacidade em seguir comportamentos mais saudáveis. Apresenta 4 afirmações, cuja concordância oscila numa escala de Likert de 7 pontos $($ de $1=$ discordo totalmente a $7=$ concordo totalmente). O total da escala é dado pela média obtida das diferentes afirmações, sendo que 


\section{Fin et al.}

quanto maior a pontuação (mais próximo de 7), maior a percepção de competência. A medida de consistência interna, utilizando o coeficiente alfa de Cronbach, foi de 0,99 .

Grau de autonomia em relação à prática de atividade física. Foi utilizado o Treatment Self-Regulation Questionnaire for Physical Activity (TSRQ-PA) (Williams, Freedman \& Deci, 1998) traduzido para o português por Marques, De Gucht, Maes, Gouveia e Leal (2012). A escala é composta por 12 itens que avaliam o grau de autonomia na regulação comportamental em relação à prática de exercícios físicos. Os participantes são apresentados a uma frase inicial "A razão pela qual eu quero fazer exercício regular é..." e classifica-se as respostas em uma escala Likert de 7 pontos $($ de $1=$ Discordo totalmente a $7=$ concordo totalmente). Sete itens representam razões controladas (2, 3, 5, 6, 7, 8 e 10) ("Sentir-me-ia envergonhado(a) se não fizesse exercício") e cinco itens representam razões mais autônomas $(1,4,9,11 \mathrm{e}$ 12) ("Pessoalmente, acredito que fazer exercício é melhor para mim"). A regulação pode ser obtida calculando-se o score-z para cada subescala e subtraindo-se o escore z de cada subescala (autônoma ou controlada). Escores mais altos indicam maior nível de autonomia no engajamento de atividade física. A medida de consistência interna, utilizando $\mathrm{o}$ coeficiente alfa de Cronbach, foi de 0,70 para os itens de regulação autônoma e 0,69 para a regulação controlada.

Escala de Satisfação com a Vida. Foi utilizada a versão em português da Satisfaction With Life Scale (SWLS) (Pavot \& Diener, 2009), e validada para a população brasileira por Bedin e Sarriera (2014) com o objetivo de avaliar o julgamento que as pessoas fazem sobre o quão satisfeitas encontram-se com suas vidas, sendo composta por 5 itens, variando de 1 (discordo totalmente) a 5 (concordo totalmente). O total da escala é dado pela média obtida das afirmações, sendo que quanto maior a pontuação (mais próximo de 5), maior a satisfação com a vida. A medida de consistência interna, utilizando o coeficiente alfa de Cronbach, foi de 0,75 .

Dermatoglifia. Para avaliar as características das impressões digitais foi determinado o uso da dermatoglifia, método proposto por Cummins e Midlo (1961), validado por Nodari Júnior, Heberle, FerreiraEmygdio e Irany-Knackfuss (2008). A partir da coleta das impressões digitais por intermédio do Leitor Dermatoglífico ${ }^{\circledR}$, a interferência do avaliador ocorre na marcação dos pontos núcleo e delta, quando, então, o software faz a identificação qualitativa da imagem identificando as figuras das impressões digitais: Arco (A), Presilha Radial (LR), Presilha Ulnar (LU), Verticilo (W), Verticilo em S (WS) desenho da mão esquerda, dedo 1 (MET1), dedo 2 (MET2), dedo 3 (MET3), dedo 4 (MET4) e dedo 5 (MET5) e, da mão direita, dedo 1 (MDT1), dedo 2 (MDT2), dedo 3 (MDT3), dedo 4 (MDT4) e dedo 5 (MDT5). O software faz também a identificação quantitativa de linhas: mão esquerda, somatório da quantidade de linhas do dedo 1 - polegar (MESQL1), mão esquerda, somatório da quantidade de linhas do dedo 2 indicador (MESQL2), mão esquerda, somatório da quantidade de linhas do dedo 3 - dedo médio (MESQL3), mão esquerda, somatório da quantidade de linhas do dedo 4 - anular (MESQL4) e mão esquerda, somatório da quantidade de linhas do dedo 5 - mínimo (MESQL5); somatório da quantidade total de linhas da mão esquerda (SQTLE); mão direita, somatório da quantidade de linhas do dedo 1 - polegar (MDSQL1), mão direita, somatório da quantidade de linhas do dedo 2 - indicador (MDSQL2), mão direita, somatório da quantidade de linhas do dedo 3 - dedo médio (MDSQL3), mão direita, somatório da quantidade de linhas do dedo 4 - anular (MDSQL4) e mão direita, somatório da quantidade de linhas do dedo 5 - mínimo (MDSQL5); somatório da quantidade total de linhas da mão direita (SQTLD); somatório da quantidade total de linhas - ambas as mãos (SQTL), gerando a planilha informatizada resultante dos dados processados.

\section{Procedimento}

Este é um estudo observacional, transversal, analítico, para investigar a associação dos constructos da teoria da autodeterminação e das características dermatoglíficas com o comportamento relacionado à prática de atividade física em mulheres que se encontram em tratamento para o câncer de mama, considerando seu histórico de atividade física. Antes da coleta de dados, houve a aprovação do setor de oncologia do Hospital Universitário Santa Terezinha, Joaçaba, Santa Catarina, Brasil, com parecer positivo do Comitê de Ética em Pesquisas em Seres Humanos da Universidade do Oeste de Santa Catarina e do Hospital Universitário Santa Terezinha (CEP- 


\section{Actividad física, motivación y dermatoglífia en mujeres con cáncer de mama}

UNOESC/HUST), sob protocolos de Certificado de Apresentação para Apreciação Ética (CAAE), número 61883216.8.0000.5367. A pesquisadora verificou a lista de pessoas em tratamento com antecedência e realizou amostragem de conveniência para os que realizam consultas e tratamento no setor de oncologia do hospital. As participantes receberam documento explicando a pesquisa e o Termo de Consentimento Livre e Esclarecido (TCLE). Após assinatura do TCLE, foi realizada a coleta das impressões digitais e aplicação do questionário. Este procedimento ocorreu em dias de consulta, sendo respeitados os horários do setor e a disponibilidade das mesmas e durou aproximadamente 25 minutos.

\section{Análise estatística}

As mulheres foram agrupadas de acordo com as respostas do histórico de prática de atividade física, se praticavam atividade física antes do diagnóstico da doença e se mantiveram a prática durante o tratamento, sendo então caracterizados os seguintes grupos: Não/Não; Não/Sim; Sim/Não; Sim/Sim.

As variáveis categóricas foram representadas pela frequência absoluta e relativa, sendo representadas por média, desvio-padrão, mediana e intervalo interquartílico (mediana [p25; p75]). Foi realizado para as variáveis quantitativas, inicialmente, o teste de normalidade de Shapiro-Wilk. A correlação de Pearson para verificar o grau de relação entre as variáveis. Para comprovar o poder preditivo das variáveis foi realizada uma análise de regressão linear múltipla hierárquica.
Para comparar as combinações das categorias das mulheres de acordo com as respostas do histórico de prática de atividade física, antes do diagnóstico da doença e durante o tratamento, utilizou-se o teste de Kruskal-Wallis, uma vez que um dos grupos apresentou tamanho (n) menor que 12 sujeitos. Estudou-se também os graus de relações entre as características dermatoglíficas e as variáveis competência percebida, grau de autonomia para a prática de atividade física nível de atividade física e satisfação com a vida. O nível de significância adotado foi de 0.05 . As análises foram realizadas no SPSS v.25.

\section{RESULTADOS}

Foram coletadas informações de 104 mulheres, com média de idade de 54 anos ( $\mathrm{DP}=11$, mín. $=25$, máx $=81$ ), atendidas no Setor de Oncologia de um hospital do meio oeste do estado de Santa Catarina, Brasil.

Quando comparadas as distribuições das variáveis percepção de competência $(p<0,001)$, regulação autônoma $(\mathrm{p}=0,002)$, nível de atividade física $(p<0,001)$ e escala de satisfação com a vida $(p=0,005)$ entre as categorias do histórico de prática de atividade física (Antes/Depois do diagnóstico) foram encontradas diferença significativas (Tabela 1). Os resultados mostraram que no grupo de mulheres que fazia atividade física antes do diagnóstico e continuou com a atividade durante o tratamento para o câncer de mama, a percepção de competência, a regulação autônoma, a satisfação com a vida e consequentemente o nível de atividade física foram maiores.

Tabela 1. Comparações das distribuições das variáveis estudadas entre as respostas do histórico de atividade fisica, antes do diagnóstico e durante o tratamento $(n=104)$

\begin{tabular}{|c|c|c|c|c|c|}
\hline & \multicolumn{4}{|c|}{ Prática Atividade Física: Antes / Depois } & \multirow{3}{*}{$\mathrm{p}$} \\
\hline & $\begin{array}{c}\text { Não/Não } \\
\mathrm{n}=20\end{array}$ & $\begin{array}{c}\text { Não/Sim } \\
n=8\end{array}$ & $\begin{array}{c}\text { Sim/Não } \\
n=31\end{array}$ & $\begin{array}{c}\operatorname{Sim} / \operatorname{Sim} \\
\mathrm{n}=45\end{array}$ & \\
\hline & $\begin{array}{c}\text { mediana } \\
{[\mathrm{P} 25 ; \mathrm{P} 75]} \\
\end{array}$ & $\begin{array}{c}\text { Mediana } \\
{[\mathrm{P} 25 ; \mathrm{P} 75]} \\
\end{array}$ & $\begin{array}{c}\text { mediana } \\
{[\mathrm{P} 25 ; \mathrm{P} 75]} \\
\end{array}$ & $\begin{array}{c}\text { mediana } \\
{[\mathrm{P} 25 ; \mathrm{P} 75]} \\
\end{array}$ & \\
\hline PCS & $2,5 \mathrm{a}[1,3 ; 4,9]$ & $5,0 \mathrm{ab}[4,5 ; 5,3]$ & $5,0 \mathrm{a}[2,0 ; 7,0]$ & $7,0 \mathrm{~b}[6,0 ; 7,0]$ & $<0,001^{*}$ \\
\hline Reg. Autônoma & $7,0 \mathrm{a}[6,7 ; 7,0]$ & $7,0 \mathrm{ab}[6,5 ; 7,0]$ & $7,0 \mathrm{a}[6,6 ; 7,0]$ & $7,0 \mathrm{~b}[7,0 ; 7,0]$ & $0,002^{*}$ \\
\hline Reg. Controlada & $3,2[2,4 ; 4,2]$ & $3,8[2,3 ; 4,4]$ & $2,7[2,6 ; 3,7]$ & $2,7[2,4 ; 3,4]$ & 0,367 \\
\hline Nível de AF & $5,0 \mathrm{a}[4,6 ; 5,8]$ & $5,9 \mathrm{ab}[5,4 ; 6,5]$ & $5,0 \mathrm{a}[4,5 ; 5,8]$ & $6,0 \mathrm{~b}[5,5 ; 6,3]$ & $<0,001^{*}$ \\
\hline Satisfação Vida & $4,5 \mathrm{ab}[4,0 ; 5,0]$ & $4,3 \mathrm{a}[3,5 ; 4,8]$ & $4,8 \mathrm{ab}[3,8 ; 5,3]$ & $5,3 \mathrm{~b}[4,5 ; 5,8]$ & $0,005^{*}$ \\
\hline
\end{tabular}




\section{Fin et al.}

Foi utilizada a regressão linear múltipla para verificar se as variáveis percepção de competência, regulação autônoma, nível de atividade física e satisfação com a vida eram preditoras da prática de atividade física antes e depois do diagnóstico de câncer. A análise resultou em um modelo estatisticamente significativo $[\mathrm{F}(2,101)=23,380 ; \mathrm{p}<0,001 ; \mathrm{R} 2=0,316]$, em que a percepção de competência $(\beta=0,457 ; \mathrm{t}=5,409 ; \mathrm{p}<$ $0,001)$ e a regulação autônoma $(\beta=0,238 ; \mathrm{t}=2,815$; $\mathrm{p}=0,006$ ) são capazes de prever a prática de atividade física.

Nas análises dermatoglíficas, 90 das 104 mulheres apresentaram todas as impressões digitais com qualidade para serem avaliadas. Quando comparadas as distribuições da quantidade de linhas para observação da dermatoglifia e as demais variáveis estudadas, não foram encontradas correlações significativas (Tabela 2).

Ao observar as variáveis dermatoglíficas dos desenhos das impressões digitais, observou-se que no polegar (MDT1, p=0,047) e indicador (MDT2, $\mathrm{p}=0,026)$ da mão direita, a distribuição de verticilos em $\mathrm{S}$ desenho (WS) foi maior nas mulheres que apresentaram regulação controlada para prática de atividade física (Tabela 3).

Tabela 2. 1Correlação entre as medidas das características dermatoglíficas e as

\begin{tabular}{ccccccccc}
\multicolumn{1}{c}{ variáveis estudadas $(n=90)$} & \multicolumn{1}{c}{ PSC } & \multicolumn{1}{c}{ Reg. Autônoma } & \multicolumn{2}{c}{ Reg. Controlada } & \multicolumn{2}{c}{ ESV } \\
& $\mathrm{r}$ & $\mathrm{p}$ & $\mathrm{r}$ & $\mathrm{p}$ & $\mathrm{r}$ & $\mathrm{p}$ & $\mathrm{r}$ & $\mathrm{p}$ \\
\hline MESQL1 & $-0,139$ & 0,193 & $-0,034$ & 0,748 & $-0,028$ & 0,794 & $-0,034$ & 0,751 \\
MESQL2 & $-0,026$ & 0,806 & 0,073 & 0,494 & $-0,020$ & 0,850 & 0,118 & 0,267 \\
MESQL3 & $-0,122$ & 0,250 & 0,033 & 0,755 & $-0,040$ & 0,705 & $-0,003$ & 0,976 \\
MESQL4 & 0,085 & 0,427 & 0,050 & 0,642 & $-0,100$ & 0,351 & $-0,021$ & 0,841 \\
MESQL5 & 0,044 & 0,682 & $-0,054$ & 0,610 & $-0,032$ & 0,763 & $-0,010$ & 0,923 \\
SQTLE & $-0,045$ & 0,676 & 0,019 & 0,860 & $-0,056$ & 0,603 & 0,026 & 0,807 \\
MDSQL1 & 0,044 & 0,681 & $-0,014$ & 0,895 & 0,036 & 0,734 & $-0,097$ & 0,366 \\
MDSQL2 & 0,026 & 0,810 & $-0,033$ & 0,757 & $-0,094$ & 0,376 & $-0,029$ & 0,789 \\
MDSQL3 & 0,002 & 0,982 & $-0,078$ & 0,464 & $-0,086$ & 0,422 & $-0,041$ & 0,703 \\
MDSQL4 & $-0,025$ & 0,813 & $-0,069$ & 0,515 & 0,009 & 0,934 & $-0,002$ & 0,987 \\
MDSQL5 & 0,035 & 0,741 & $-0,091$ & 0,394 & $-0,049$ & 0,649 & $-0,047$ & 0,658 \\
SQTLD & 0,023 & 0,831 & $-0,078$ & 0,467 & $-0,052$ & 0,624 & $-0,059$ & 0,583 \\
SQTL & $-0,014$ & 0,898 & $-0,028$ & 0,795 & $-0,057$ & 0,594 & $-0,014$ & 0,893
\end{tabular}

Nota. Correlação de Pearson (valor-p). PCS: Percepção de Competência; Reg.: Regulação; ESV: Escala de Satisfação com a Vida. 


\section{Actividad física, motivación y dermatoglífia en mujeres con cáncer de mama}

Tabela 3. Comparações das distribuições das variáveis dermatoglíficas e as variáveis estudadas

\begin{tabular}{|c|c|c|c|c|c|c|}
\hline & & \multirow{2}{*}{$\mathrm{n}$} & PCS & \multicolumn{2}{|c|}{ Regulação } & \multirow{2}{*}{$\begin{array}{c}\text { ESV } \\
\text { med }[\mathrm{P} 25 ; \mathrm{P} 75]\end{array}$} \\
\hline & & & med [P25; P75] & $\begin{array}{c}\text { Autônoma } \\
\text { med [P25; P75] }\end{array}$ & $\begin{array}{c}\text { Controlada } \\
\text { med }[\mathrm{P} 25 ; \mathrm{P} 75]\end{array}$ & \\
\hline \multirow[t]{6}{*}{ MET1 } & $\mathrm{A}$ & 8 & $6,5[5,5 ; 7,0]$ & $7,0[6,9 ; 7,0]$ & $2,6[2,6 ; 3,4]$ & $4,3[3,6 ; 5,1]$ \\
\hline & LR & 3 & $6,5[6,0 ; 7,0]$ & $7,0[7,0 ; 7,0]$ & $2,3[2,1 ; 3,9]$ & $6,0[5,0 ; 6,3]$ \\
\hline & LU & 53 & $5,0[2,3 ; 7,0]$ & $7,0[6,8 ; 7,0]$ & $2,7[2,4 ; 3,6]$ & $4,8[3,8 ; 5,3]$ \\
\hline & W & 10 & $4,5[3,8 ; 7,0]$ & $6,9[6,2 ; 7,0]$ & $2,7[2,4 ; 4,1]$ & $4,4[3,8 ; 5,3]$ \\
\hline & WS & 16 & $6,3[5,0 ; 7,0]$ & $7,0[7,0 ; 7,0]$ & $4,0[2,9 ; 5,1]$ & $5,0[4,5 ; 5,4]$ \\
\hline & $\mathrm{p}$ & & 0,209 & 0,171 & 0,131 & 0,216 \\
\hline \multirow[t]{6}{*}{ MET2 } & A & 7 & $7,0[5,0 ; 7,0]$ & $7,0[7,0 ; 7,0]$ & $3,3[2,7 ; 4,0]$ & $5,0[4,5 ; 5,8]$ \\
\hline & LR & 19 & $6,0[4,0 ; 7,0]$ & $7,0[7,0 ; 7,0]$ & $2,7[2,6 ; 4,3]$ & $4,3[3,8 ; 5,3]$ \\
\hline & LU & 34 & $5,0[3,0 ; 6,8]$ & $7,0[6,8 ; 7,0]$ & $2,7[2,3 ; 3,4]$ & $4,8[3,8 ; 5,5]$ \\
\hline & W & 19 & $5,0[1,0 ; 7,0]$ & $7,0[6,8 ; 7,0]$ & $3,6[2,3 ; 4,7]$ & $4,5[3,5 ; 5,3]$ \\
\hline & WS & 11 & $5,0[4,0 ; 7,0]$ & $7,0[7,0 ; 7,0]$ & $2,9[2,4 ; 3,6]$ & $5,0[4,3 ; 5,3]$ \\
\hline & $\mathrm{p}$ & & 0,511 & 0,460 & 0,328 & 0,652 \\
\hline \multirow[t]{6}{*}{ MET3 } & A & 8 & $6,5[3,5 ; 7,0]$ & $7,0[7,0 ; 7,0]$ & $3,4[2,4 ; 4,4]$ & $5,1[4,5 ; 6,3]$ \\
\hline & LR & 1 & $6,0[6,0 ; 6,0]$ & $7,0[7,0 ; 7,0]$ & $2,7[2,7 ; 2,7]$ & $3,5[3,5 ; 3,5]$ \\
\hline & LU & 68 & $5,0[4,0 ; 7,0]$ & $7,0[6,8 ; 7,0]$ & $2,7[2,4 ; 3,8]$ & $4,8[3,9 ; 5,4]$ \\
\hline & $\mathrm{W}$ & 5 & $5,0[5,0 ; 6,8]$ & $7,0[7,0 ; 7,0]$ & $3,7[2,7 ; 5,0]$ & $4,8[4,5 ; 5,0]$ \\
\hline & WS & 8 & $3,5[1,0 ; 6,5]$ & $7,0[6,9 ; 7,0]$ & $3,1[2,4 ; 4,1]$ & $5,1[4,0 ; 5,6]$ \\
\hline & $\mathrm{p}$ & & 0,688 & 0,847 & 0,749 & 0,418 \\
\hline \multirow[t]{6}{*}{ MET4 } & A & 2 & $4,5[2,0 ; 7,0]$ & $7,0[7,0 ; 7,0]$ & $2,6[1,7 ; 3,6]$ & $5,6[5,0 ; 6,3]$ \\
\hline & LR & 3 & $5,0[5,0 ; 7,0]$ & $7,0[6,8 ; 7,0]$ & $3,1[2,6 ; 5,0]$ & $4,3[4,0 ; 5,3]$ \\
\hline & LU & 62 & $5,0[3,0 ; 7,0]$ & $7,0[6,8 ; 7,0]$ & $2,8[2,4 ; 3,7]$ & $4,8[3,8 ; 5,5]$ \\
\hline & W & 18 & $6,3[5,0 ; 7,0]$ & $7,0[7,0 ; 7,0]$ & $2,7[2,4 ; 3,3]$ & $5,0[4,5 ; 5,3]$ \\
\hline & WS & 5 & $1,0[1,0 ; 1,0]$ & $7,0[7,0 ; 7,0]$ & $5,0[4,7 ; 5,1]$ & $4,5[4,3 ; 4,8]$ \\
\hline & $\mathrm{p}$ & & 0,116 & 0,807 & 0,122 & 0,470 \\
\hline \multirow[t]{5}{*}{ MET5 } & A & 4 & $2,5[1,5 ; 4,8]$ & $7,0[7,0 ; 7,0]$ & $2,5[1,9 ; 3,4]$ & $4,8[3,8 ; 5,5]$ \\
\hline & LU & 75 & $5,8[4,0 ; 7,0]$ & $7,0[6,8 ; 7,0]$ & $2,7[2,4 ; 3,7]$ & $4,8[4,0 ; 5,5]$ \\
\hline & W & 6 & $5,0[5,0 ; 7,0]$ & $7,0[7,0 ; 7,0]$ & $2,7[2,7 ; 4,4]$ & $4,9[4,3 ; 5,3]$ \\
\hline & WS & 5 & $5,0[4,0 ; 6,3]$ & $7,0[6,8 ; 7,0]$ & $2,7[2,4 ; 4,3]$ & $5,0[3,8 ; 5,0]$ \\
\hline & $\mathrm{p}$ & & 0,448 & 0,262 & 0,817 & 0,994 \\
\hline \multirow[t]{5}{*}{ MDT1 } & A & 2 & $6,3[6,0 ; 6,5]$ & $7,0[7,0 ; 7,0]$ & $2,2 \mathrm{a}[2,1 ; 2,3]$ & $6,1[6,0 ; 6,3]$ \\
\hline & LU & 57 & $5,0[3,8 ; 7,0]$ & $7,0[6,8 ; 7,0]$ & $2,7 \mathrm{ab}[2,6 ; 3,6]$ & $4,8[3,8 ; 5,3]$ \\
\hline & W & 8 & $5,0[3,0 ; 6,5]$ & $7,0[6,4 ; 7,0]$ & $2,6 \mathrm{ab}[2,1 ; 2,9]$ & $4,6[4,3 ; 5,3]$ \\
\hline & WS & 23 & $5,0[2,0 ; 7,0]$ & $7,0[7,0 ; 7,0]$ & $3,7 \mathrm{~b}[2,6 ; 4,9]$ & $5,0[4,3 ; 5,8]$ \\
\hline & $\mathrm{p}$ & & 0,924 & 0,514 & $\mathbf{0 , 0 4 7 ^ { * }}$ & 0,107 \\
\hline \multirow[t]{6}{*}{ MDT2 } & A & 4 & $6,0[4,9 ; 7,0]$ & $6,9[6,7 ; 7,0]$ & 3,5ab $[3,2 ; 3,9]$ & $5,0[4,6 ; 5,8]$ \\
\hline & LR & 14 & $6,0[2,3 ; 7,0]$ & $7,0[6,6 ; 7,0]$ & $2,7 \mathrm{ab}[2,6 ; 3,3]$ & $3,5[3,0 ; 4,8]$ \\
\hline & LU & 42 & $5,0[3,8 ; 7,0]$ & $7,0[7,0 ; 7,0]$ & $2,7 \mathrm{ab}[2,4 ; 3,7]$ & $5,0[4,3 ; 5,5]$ \\
\hline & W & 16 & $5,4[4,0 ; 6,5]$ & $7,0[6,8 ; 7,0]$ & $2,6 \mathrm{a}[2,3 ; 3,4]$ & $4,6[3,8 ; 5,5]$ \\
\hline & WS & 14 & $5,0[1,5 ; 7,0]$ & $7,0[7,0 ; 7,0]$ & $4,7 \mathrm{~b}[3,3 ; 5,3]$ & $5,0[4,5 ; 5,3]$ \\
\hline & $\mathrm{p}$ & & 0,893 & 0,691 & $\mathbf{0 , 0 2 6}{ }^{*}$ & 0,063 \\
\hline \multirow[t]{6}{*}{ MDT3 } & A & 5 & $6,0[5,0 ; 7,0]$ & $7,0[7,0 ; 7,0]$ & $3,6[2,3 ; 4,3]$ & $5,0[5,0 ; 6,3]$ \\
\hline & LR & 2 & $5,5[4,0 ; 7,0]$ & $7,0[7,0 ; 7,0]$ & $2,6[2,6 ; 2,7]$ & $5,4[5,0 ; 5,8]$ \\
\hline & LU & 74 & $5,0[3,8 ; 7,0]$ & $7,0[6,8 ; 7,0]$ & $2,7[2,4 ; 3,9]$ & $4,6[3,8 ; 5,3]$ \\
\hline & W & 4 & $3,6[1,0 ; 6,5]$ & $7,0[7,0 ; 7,0]$ & $2,5[1,6 ; 3,7]$ & $5,1[3,5 ; 5,9]$ \\
\hline & WS & 5 & $5,0[5,0 ; 7,0]$ & $7,0[7,0 ; 7,0]$ & $3,3[2,7 ; 3,7]$ & $5,3[5,0 ; 5,3]$ \\
\hline & $\mathrm{p}$ & & 0,832 & 0,336 & 0,854 & 0,158 \\
\hline \multirow[t]{6}{*}{ MDT4 } & A & 1 & $5,0[5,0 ; 5,0]$ & $7,0[7,0 ; 7,0]$ & $6,6[6,6 ; 6,6]$ & $5,0[5,0 ; 5,0]$ \\
\hline & LR & 1 & $5,0[5,0 ; 5,0]$ & $6,8[6,8 ; 6,8]$ & $3,1[3,1 ; 3,1]$ & $5,3[5,3 ; 5,3]$ \\
\hline & LU & 54 & $5,9[3,0 ; 7,0]$ & $7,0[6,8 ; 7,0]$ & $2,7[2,4 ; 3,7]$ & $4,8[3,8 ; 5,8]$ \\
\hline & W & 26 & $5,0[4,0 ; 7,0]$ & $7,0[6,8 ; 7,0]$ & $2,8[2,6 ; 4,1]$ & $4,6[4,3 ; 5,3]$ \\
\hline & WS & 8 & $6,0[1,0 ; 7,0]$ & $7,0[7,0 ; 7,0]$ & $2,5[1,9 ; 3,6]$ & $5,0[4,6 ; 5,6]$ \\
\hline & $\mathrm{p}$ & & 0,995 & 0,556 & 0,346 & 0,775 \\
\hline \multirow[t]{6}{*}{ MDT5 } & A & 2 & $3,8[1,0 ; 6,5]$ & $7,0[7,0 ; 7,0]$ & $3,1[2,1 ; 4,0]$ & $5,3[4,5 ; 6,0]$ \\
\hline & LR & 1 & $7,0[7,0 ; 7,0]$ & $7,0[7,0 ; 7,0]$ & $1,9[1,9 ; 1,9]$ & $4,5[4,5 ; 4,5]$ \\
\hline & LU & 77 & $5,5[4,0 ; 7,0]$ & $7,0[6,8 ; 7,0]$ & $2,7[2,6 ; 3,7]$ & $4,8[4,0 ; 5,3]$ \\
\hline & W & 6 & $5,0[2,8 ; 6,3]$ & $6,9[6,8 ; 7,0]$ & $2,6[2,0 ; 3,1]$ & $5,1[5,0 ; 6,0]$ \\
\hline & WS & 4 & $3,0[1,0 ; 6,0]$ & $7,0[7,0 ; 7,0]$ & $4,6[3,9 ; 4,9]$ & $4,8[3,4 ; 5,6]$ \\
\hline & $\mathrm{p}$ & & 0,482 & 0,444 & 0,082 & 0,702 \\
\hline
\end{tabular}

Nota. Teste de Kruskal-Wallis. Letras distintas representam distribuições estatisticamente diferentes 


\section{Fin et al.}

\section{DISCUSSÃO}

Este estudo tem o objetivo de investigar a associação dos constructos da teoria da autodeterminação e das características dermatoglíficas sobre o comportamento relacionado à prática de atividade física em mulheres que se encontram em tratamento para o câncer de mama, considerando seu histórico de atividade física.

De maneira geral, sabe-se que as pessoas que estabelecem uma maior importância para a saúde e competência para a prática de atividade física tem uma maior probabilidade de cumprir as recomendações mínimas para manter os níveis de atividade física (Romo, Muñoz, González \& Adam, 2018; Pardo, Castrillón, Pedreño \& Moreno-Murcia, 2014). Neste estudo, quando avaliadas as mulheres que estão em tratamento para o câncer de mama, observa-se que a percepção de competência e a regulação autônoma demonstraram ser características importantes para a manutenção da prática de atividade física durante o tratamento para o câncer de mama. Estes resultados são semelhantes aos encontrados na literatura em pessoas com diabetes, que apresentaram a percepção de competência e a regulação autônoma mais fortemente associada ao comportamento relacionado a uma dieta saudável (Oumrait et al., 2020). Também foi comprovada a relação da competência e a prática de atividade física entre sobreviventes de câncer colorretal (Kim et al., 2020) e a importância da regulação autônoma para o comportamento relacionado à prática de atividade física em pessoas com artrite reumatoide (Fenton et al., 2020).

A competência mostrou ter um efeito positivo associação com atividade física, também em outros estudos. Por exemplo, uma meta-análise sobre a TAD no domínio da saúde descobriu que a competência era a variável que mais contribuiu para melhorias nos níveis de prática (Fortier, Duda, Guerin, Teixeira, 2012), sendo um forte preditor para a atividade física (Vlachopoulos, Kaperoni, Moustaka, 2011). De acordo com Deci e Ryan (2000), competência é a percepção subjetiva de habilidades que interagem efetivamente com o meio ambiente. Isso sugere que, se o paciente se percebe capaz de fazê-lo, ele pode realizar efetivamente e por mais tempo a atividade física. A competência pode levar a uma resposta comportamental positiva, melhorando o controle sobre o comportamento, aumentando o interesse e o esforço para realizar atividades físicas, e levando à adoção de hábitos mais saudáveis. Além disso, um estudo especificamente com sobreviventes de câncer de mama e próstata (Mosher, Lipkus, Sloane, Snyder, Lobach \& Demark-Wahnefried, 2013) também descobriu que a competência era o preditor mais forte sobre a atividade física, fornecendo evidências adicionais para a importância desta associação.

Os resultados sobre a regulação autônoma para a prática de atividade física também têm implicações na maneira como as discussões sobre estes comportamentos são direcionados em pessoas que se encontram no tratamento para o câncer. Especificamente, os resultados podem sugerir que o suporte à autonomia oferecido por profissionais que interagem com pessoas em tratamento é uma das razões para promover a internalização e mudança positiva relacionada ao comportamento para atividade física. Uma intervenção com pessoas que apresentam artrite reumatoide demonstrou a importância do suporte à autonomia recebido pelos profissionais, para a adesão ao exercício (Oumrait et al., 2020), destacando o valor potencial das interações de apoio à autonomia como estratégias de intervenção para promover atividade física no tratamento de doenças e o papel da motivação autônoma como um fator que pode ser modificável e determinante tendo como suporte os princípios teóricos da TAD.

Ao aumentar os sentimentos de competência e autonomia da pessoa, podemos ter também o aumento nos níveis de atividade física e consequentemente melhora na satisfação com a vida (Aguiñaga, Ehlers, Cosman, Severson, Kramer \& McAuley, 2018). Além disso, neste estudo, os resultados corroboram esse efeito na população com câncer de mama, mostrando que, a prática de atividade física era maior nas mulheres que tinham maior percepção de competência e regulação autônoma, assim como as taxas de satisfação com a vida eram superiores. Nesse sentido, os sentimentos de competência e autonomia, permitem que a pessoa realize atividades físicas, por considerar que este comportamento faz parte de um estilo de vida saudável (Fortier, Duda, Guerin \& Teixeira, 2012), impactando positivamente no seu bem-estar global, físico e psicológico, com razões sobre o efeito dessas variáveis sobre a satisfação com a vida da pessoa.

Considerando as características inatas, que podem ser fatores determinantes sobre atitudes relacionadas à saúde, foram observadas marcas nas impressões 


\section{Actividad física, motivación y dermatoglífia en mujeres con cáncer de mama}

digitais que se diferenciam entre os grupos investigados, sendo que no polegar e indicador da mão direita, a distribuição de verticilos em $\mathrm{S}$ desenho (WS) foi maior nas mulheres que apresentaram regulação controlada para prática de atividade física. Nestas mulheres, a regulação controlada gera comportamentos de pressão externa, indicando o sentido oposto a regulação autônoma, havendo neste caso uma dificuldade em perceber a importância da prática da atividade física por si só. Nas razões controladas, existe a necessidade de haver uma pressão, interna ou externa, para a realização de certos comportamentos, o que é pouco proveitoso, fazendo com que haja maior desistência e resultados negativos diante do objetivo (Vansteenkiste, Williams \& Resnicow, 2012).

É importante destacar que existem dificuldades em coletar as impressões digitais das pessoas que se encontram em tratamento para o câncer, pois um dos efeitos colaterais da quimioterapia e radioterapia é o ressecamento e descamação da pele, o que acaba afetando a qualidade das impressões digitais, resultando em perdas na amostra. Para os resultados relacionados às características dermatoglíficas, uma das limitações deste estudo foi o tamanho amostral, pois a distribuição se torna pequena em cada categoria observada. Sendo assim, mesmo apresentando diferenças significativas, sugere-se que estes resultados sejam replicados em amostras maiores.

Ainda como limitações do estudo, a amostra compreende mulheres com uma grande dispersão de idade (25 a 81 anos), neste sentido, estudos futuros devem considerar uma amostra mais homogênea, uma vez que fatores psicológicos e ambientais desempenham um papel relevante na promoção da participação em exercícios físicos em idosos ou em pessoas mais jovens (Martín-Moya, Ruiz-Montero, Garcia \& Leeson, 2020).

\section{CONCLUSÕES}

A percepção de competência e a regulação autônoma podem ser preditoras da prática de atividade física de mulheres em tratamento para o câncer de mama. Sendo observado que no grupo de mulheres que realizavam atividade física antes do diagnóstico de câncer e continuaram durante $\mathrm{o}$ tratamento, a percepção de competência, a regulação autônoma, a satisfação com a vida e o nível de atividade física era maior. As características dermatoglíficas encontradas podem ser um indicativo de uma marca que pode influenciar a regulação comportamental em relação à prática de atividades físicas, porém mais estudos são necessários para verificar como essas variáveis se comportam no âmbito do câncer de mama.

\section{APLICAÇÕES PRÁTICAS}

Embora existam estudos com populações análogas, não conhecemos trabalhos que relacionem os constructos da TAD com a prática de atividade física, a satisfação com a vida e as variáveis dermatoglíficas, na população que se encontra em tratamento para o câncer de mama. Este estudo é, portanto, um primeiro passo para desenvolver futuros desenhos de investigação e intervenção que auxiliem a compreensão da influência da autodeterminação e das características dermatoglíficas sobre o comportamento para a prática da atividade física e a satisfação com a vida em pessoas com câncer de mama.

Recomenda-se a realização de estudos randomizados controlados nos quais sejam verificadas as relações entre o nível de atividade física das pessoas com câncer, os constructos da TAD e a satisfação com a vida. Seria também interessante verificar, em futuros estudos, a influência da idade nos resultados obtidos.

Este trabalho é novo pelas informações que fornece sobre a motivação das pessoas com câncer de mama para a prática de atividade física durante o tratamento, abordando as relações entre a competência, autonomia, os níveis de atividade física e satisfação com a vida. Provavelmente, mais estudos são necessários para verificar como essas variáveis se comportam em longo prazo em pessoas com câncer de mama, para que seja possível obter um modelo que auxilie profissionais na implementação de desenhos de intervenção que melhorem o nível de atividade física e a satisfação com os vida dessas pessoas.

Este estudo teve suporte financeiro da Fundação de Amparo à Pesquisa e Inovação do Estado de Santa Catarina (FAPESC), e de Bolsa de Estudos do Programa UNIEDU Pós-graduação, do Estado de Santa Catarina, Brasil. 


\section{Fin et al.}

\section{REFERENCIAS}

1. Abramova, T. F., Nikitina, T. M., Izaak, S. I., \& Kochetkova, N. I. (2000). Asymmetry of signs of finger dermatoglyphics, physical potential and physical qualities of a man. Morfologiia (Saint Petersburg, Russia), 118(5), 56-59.

2. Aguiñaga, S., Ehlers, D. K., Cosman, J., Severson, J., Kramer, A. F., \& McAuley, E. (2018). Effects of physical activity on psychological well-being outcomes in breast cancer survivors from prediagnosis to posttreatment survivorship. Psycho-oncology, 27(8), 1987-1994. https://doi.org/10.1002/pon.4755

3. Ainsworth, B. E., Haskell, W. L., Whitt, M. C., Irwin, M. L., Swartz, A. M., Strath, S. J., ... \& Leon, A. S. (2000). Compendium of physical activities: an update of activity codes and MET intensities. Medicine and science in sports and exercise, $\quad 32(9 \quad$ Suppl), S498-S504. https://doi.org/10.1097/00005768-20000900100009

4. Akbarova, S. N. (2018). Dermatogliphics can be as method of behavior genetics. Education Sciences \& Psychology, 50(4).

5. Baecke, J. A., Burema, J., Frijters, J. E. (1982). A short questionnaire for the measurement of habitual physical activity in epidemiological studies. Am J Clin Nutr., 36(5), 936-942. doi:10.1093/ajen/36.5.936.

https://doi.org/10.1093/ajen/36.5.936

6. Bedin, L. M., \& Sarriera, J. C. (2014). Propriedades psicométricas das escalas de bemestar: PWI, SWLS, BMSLSS e CAS. Avaliação Psicologica: Interamerican Journal of Psychological Assessment, 13(2), 213-225.

7. Bierman, H. R., Faith, M. R., \& Stewart, M. E. (1988). Digital dermatoglyphics in mammary cancer. Cancer investigation, 6(1), 15-27. https://doi.org/10.3109/07357908809077025

8. Bouchard Jr, T. J., \& McGue, M. (1990). Genetic and rearing environmental influences on adult personality: An analysis of adopted twins reared apart. Journal of Personality, 58(1), 263-292. https://doi.org/10.1111/j.14676494.1990.tb00916.x
9. Buffart, L. M., Galvão, D. A., Brug, J., Chinapaw, M. J. M., \& Newton, R. U. (2014). Evidencebased physical activity guidelines for cancer survivors: current guidelines, knowledge gaps and future research directions. Cancer treatment reviews, 40(2), 327-340. doi: 10.1016/j.ctrv.2013.06.007.

https://doi.org/10.1016/j.ctrv.2013.06.007

10. Cummins, H., \& Midlo, C. (1961). Finger prints, palms and soles: an introduction to dermatoglyphics (Vol. 319). New York: Dover Publications.

11. Chatzisarantis, N. L., Hagger, M. S., Kamarova, S., \& Kawabata, M. (2012). When effects of the universal psychological need for autonomy on health behaviour extend to a large proportion of individuals: a field experiment. British journal of health psychology, 17(4), 785-797. https://doi.org/10.1111/j.20448287.2012.02073.x

12. de Rezende, L. F. M., de Sá, T. H., Markozannes, G., Rey-López, J. P., Lee, I. M., Tsilidis, K. K., ... \& Eluf-Neto, J. (2018). Physical activity and cancer: an umbrella review of the literature including 22 major anatomical sites and 770000 cancer cases. British journal of sports medicine, 52(13), 826-833. https://doi.org/10.1136/bjsports2017-098391

13. Deci, E. L., \& Ryan, R. M. (1991). A motivational approach to self: Integration in personality. In R. A. Dienstbier (Ed.), Current theory and research in motivation, Vol. 38. Nebraska Symposium on Motivation, 1990: Perspectives on motivation ( $\mathrm{p}$. 237-288). University of Nebraska Press.

14. Deci, E. L., \& Ryan, R. M. (2000). The "what" and "why" of goal pursuits: human needs and the self-determination of behaviour. Psychological Inquiry, 11(4), 227-268. https://doi.org/10.1207/S15327965PLI1104_01

15. Deci, E. L., \& Ryan, R. M. (2017). SelfDetermination Theory: Basic psychological meeds in motivation, development and wellness. New York, NY: The Guilford Press.

16. Duda, J. L., Williams, G. C., Ntoumanis, N., Daley, A., Eves, F. F., Mutrie, N., ... \& Jolly, K. (2014). Effects of a standard provision versus an autonomy supportive exercise referral programme 


\section{Actividad física, motivación y dermatoglífia en mujeres con cáncer de mama}

on physical activity, quality of life and well-being indicators: a cluster randomised controlled trial. International Journal of Behavioural Nutrition and Physical Activity, 11(1), 10. https://doi.org/10.1186/1479-5868-11-10

17. Fenton, S. A., Veldhuijzen van Zanten, J. J., Metsios, G. S., Rouse, P. C., Yu, C. A., Ntoumanis, N., ... \& Duda, J. L. (2020). Testing a self-determination theory-based process model of physical activity behaviour change in rheumatoid arthritis: results of a randomized controlled trial. Translational Behavioural Medicine, 2, 1-12. https://doi.org/10.1093/tbm/ibaa022

18. Florindo, A. A., Latorre, M. R. D. O. (2003). Validação do questionário de Baecke de avaliação da atividade física habitual em homens adultos. Revista Brasileira de Medicina do Esporte, 9, 1218.

19. Fortier, M. S., Duda, J. L., Guerin, E., \& Teixeira, P. J. (2012). Promoting physical activity: development and testing of self-determination theory-based interventions. International Journal of Behavioural Nutrition and Physical Activity, 9(1), 20. https://doi.org/10.1186/1479-5868-9-20

20. Gillison, F. B., Rouse, P., Standage, M., Sebire, S. J., \& Ryan, R. M. (2019). A meta-analysis of techniques to promote motivation for health behaviour change from a self-determination theory perspective. Health psychology review, 13(1), 110-130. https://doi.org/10.1080/17437199.2018.1534071

21. Halvari, H., Healey, J., Olafsen, A. H., Byrkjeland, R., Deci, E. L., \& Williams, G. C. (2017). Physical activity and motivational predictors of changes in health behavior and health among DM 2 and CAD patients. Scandinavian Journal of Medicine \& Science in Sports, 27(11), 1454-1469. https://doi.org/10.1111/sms.12757

22. Husted, G. R., Thorsteinsson, B., Esbensen, B. A., Gluud, C., Winkel, P., Hommel, E., \& Zoffmann, V. (2014). Effect of guided self-determination youth intervention integrated into outpatient visits versus treatment as usual on glycemic control and life skills: a randomized clinical trial in adolescents with type 1 diabetes. Trials, 15(1), 321. https://doi.org/10.1186/1745-6215-15-321
23. Ito, P. C. P., \& Guzzo, R. S. L. (2002). Diferenças individuais: temperamento e personalidade; importância da teoria. Estudos de Psicologia (Campinas), 19(1), 91-100. https://doi.org/10.1590/S0103166X2002000100008

24. Kahn, H. S., Graff, M., Stein, A. D., \& Lumey, L. H. (2009). A fingerprint marker from early gestation associated with diabetes in middle age: the Dutch Hunger Winter Families Study. International Journal of epidemiology, 38(1), 101109. https://doi.org/10.1093/ije/dyn158

25. Kaur, H. (2019). Co-relation of Dermatoglyphics with Lifestyle Disorders: A Review. The Indian Practitioner, 72(08), 30-33.

26. Khandelwal, R., Mittal, A., Saijanani, S., Tuteja, A., Bansal, A., Bhatnagar, D., \& Saxena, S. (2007). Qualitative and quantitative dermatoglyphic traits in patients with breast cancer: a prospective clinical study. BMC cancer, 7(1), 1-5. https://doi.org/10.1186/1471-2407-7-44

27. Kim, K. A., Chu, S. H., Oh, E. G., Shin, S. J., Jeon, J. Y., \& Lee, Y. J. (2020). Autonomy is not but competence and relatedness are associated with physical activity among colorectal cancer survivors. Supportive Care in Cancer, 1-9.

28. Kumari, K. L., Babu, V. S., \& Kumar, S. V. (2014). Dermatoglyphics and its relation to intelligence levels of young students. IOSR Journal of Dental and Medical Sciences, 13(5), 13. https://doi.org/10.9790/0853-13520103

29. Séguin Leclair, C., Lebel, S., \& Westmaas, J. L. (2021). Can Physical Activity and Healthy Diet Help Long-Term Cancer Survivors Manage their Fear of Recurrence?. Frontiers in Psychology, 12, 2210. https://doi.org/10.3389/fpsyg.2021.647432

30. Livingston, P. M., Craike, M. J., Salmon, J., Courneya, K. S., Gaskin, C. J., Fraser, S. F., ... \& ENGAGE Uro-Oncology Clinicians' Group. (2015). Effects of a clinician referral and exercise program for men who have completed active treatment for prostate cancer: a multicenter cluster randomized controlled trial (ENGAGE). Cancer, 121(15), 2646-2654. https://doi.org/10.1002/cncr.29385

31. Marques, M., De Gucht, V., Maes, S., Gouveia, M. J., \& Leal, I. (2012). Psychometric properties 


\section{Fin et al.}

of the Portuguese version of the treatment selfregulation questionnaire for physical activity (TSRQ-PA). Psychology, Community \& Health, 1(2), 212-220. https://doi.org/10.5964/pch.v1i2.32

32. Martín-Moya, R., Ruiz-Montero, P. J., García, E. R., \& Leeson, G. (2020). Psychological and environmental factors for older adults to exercise: A systematic review. Revista de Psicología del Deporte, 29(2), 93-104.

33. Mestre, S., Pais Ribeiro, J. (2008). Adaptação de três questionários para a população portuguesa baseados na teoria de autodeterminação. In Actas do $7^{\circ}$ congresso nacional de psicologia da saúde, 623-625.

34. Mosher, C. E., Lipkus, I., Sloane, R., Snyder, D. C., Lobach, D. F., \& Demark-Wahnefried, W. (2013). Long-term outcomes of the FRESH START trial: exploring the role of self-efficacy in cancer survivors' maintenance of dietary practices and physical activity. Psycho-oncology, 22(4), 876-885. https://doi.org/10.1002/pon.3089

35. Neilson, H. K., Farris, M. S., Stone, C. R., Vaska, M. M., Brenner, D. R., \& Friedenreich, C. M. (2017). Moderate-vigorous recreational physical activity and breast cancer risk, stratified by menopause status: a systematic review and metaanalysis. Menopause, 24(3), 322-344. https://doi.org/10.1097/GME.0000000000000745

36. Ng, J. Y., Ntoumanis, N., Thøgersen-Ntoumani, C., Deci, E. L., Ryan, R. M., Duda, J. L., \& Williams, G. C. (2012). Self-determination theory applied to health contexts: A meta-analysis. Perspectives on Psychological Science, 7(4), 325340. https://doi.org/10.1177/1745691612447309

37. Nodari Junior, R. J., \& Fin, G. (2016). Dermatoglifia: impressões digitais como marca genética e de desenvolvimento fetal. Joaçaba. Ed. Unoesc.

38. Nodari-Junior, R. J., Heberle, A., FerreiraEmygdio, R., \& Irany-Knackfuss, M. (2008). Impressões digitais para diagnóstico em saúde: validação de protótipo de escaneamento informatizado. Revista de Salud Pública, 10, 767776. https://doi.org/10.1590/S012400642008000500009
39. Ntoumanis, N., Ng, J. Y., Prestwich, A., Quested, E., Hancox, J. E., Thøgersen-Ntoumani, C., ... \& Williams, G. C. (2020). A meta-analysis of selfdetermination theory-informed intervention studies in the health domain: effects on motivation, health behaviour, physical, and psychological health. Health Psychology Review, 1-31.

https://doi.org/10.1080/17437199.2020.1718529

40. Oumrait, N. G., Daivadanam, M., Absetz, P., Guwatudde, D., Berggreen-Clausen, A., Alvesson, H. M., ... \& Sidney Annerstedt, K. (2020). Can Self-Determination Explain Dietary Patterns Among Adults at Risk of or with Type 2 Diabetes? A Cross-Sectional Study in SocioEconomically Disadvantaged Areas in Stockholm. Nutrients, 12(3), 620 . https://doi.org/10.3390/nu12030620

41. Pardo, P. M., Castrillón, F. O., Pedreño, N. B., \& Moreno-Murcia, J. A. (2014). Motivación autodeterminada en adultos mayores practicantes de ejercicio físico. Cuadernos de Psicología del Deporte, 14(3), 149-156. https://doi.org/10.4321/S1578 84232014000300016

42. Pavot, W., \& Diener, E. (2009). Review of the satisfaction with life scale. In Assessing wellbeing (pp. 101-117). Springer, Dordrecht. https://doi.org/10.1007/978-90-481-2354-4_5

43. Rock, C. L., Doyle, C., Demark-Wahnefried, W., Meyerhardt, J., Courneya, K. S., Schwartz, A. L., ... \& Byers, T. (2012). Nutrition and physical activity guidelines for cancer survivors. CA: a cancer journal for clinicians, 62(4), 242-274. https://doi.org/10.3322/caac.21142

44. Romero, S. A., Li, Q. S., \& Mao, J. J. (2017). Factors and barriers associated with changes in physical activity after cancer diagnosis. Journal of clinical oncology, 35(5), 162-162. https://doi.org/10.1200/JCO.2017.35.5_suppl.162

45. Romo, G. R., Pla, R. M., Muñoz, M. G., González, C. M. T., \& Adan, E. L. (2018). Motivos para la práctica de actividad física durante el tiempo libre y su relación con el cumplimento de las recomendaciones. Cuadernos de Psicología del Deporte, 18(1), 183-194. 


\section{Actividad física, motivación y dermatoglífia en mujeres con cáncer de mama}

46. Ryan, R. M., \& Deci, E. L. (2000). Selfdetermination theory and the facilitation of intrinsic motivation, social development, and well-being. American psychologist, 55(1), 68. https://doi.org/10.1037/0003-066X.55.1.68

47. Sariri, E., Kashanian, M., Vahdat, M., \& Yari, S. (2012). Comparison of the dermatoglyphic characteristics of women with and without breast cancer. European Journal of Obstetrics \& Gynecology and Reproductive Biology, 160(2), 201-204.

https://doi.org/10.1016/j.ejogrb.2011.11.001

48. Schmitz, K. H., Courneya, K. S., Matthews, C., Demark-Wahnefried, W., Galvão, D. A., Pinto, B. M., ... \& Schneider, C. M. (2010). American College of Sports Medicine roundtable on exercise guidelines for cancer survivors. Medicine \& Science in Sports \& Exercise, 42(7), 14091426. https://doi.org/10.1249/MSS.0b013e3181e0c112

49. Serebrennikova, O., Gunas, V., Klimas, L., Ocheretna, N., \& Shayuk, A. (2019). Predictive assessment of the association of dermatoglyphic indicators with indicators of personality traits, established by factor analysis. Reports of Morphology, 25(1), 12-18. https://doi.org/10.31393/morphology-journal2019-25(1)-02

50. Speck, R. M., Courneya, K. S., Mâsse, L. C., Duval, S., \& Schmitz, K. H. (2010). An update of controlled physical activity trials in cancer survivors: a systematic review and meta-analysis. Journal of Cancer Survivorship, 4(2), 87-100. https://doi.org/10.1007/s11764-009-0110-5

51. Suresch, R., \& Padmalatha, K. (2019). Correlation of dermatoglyphics with emotional intelligence amongst medical students. Int J Anat Res, 7(4.3), 7140-48. https://doi.org/10.16965/ijar.2019.327

52. Ungar, N., Wiskemann, J., \& Sieverding, M. (2016). Physical activity enjoyment and selfefficacy as predictors of cancer Patients' physical activity level. Frontiers in Psychology, 7, 898. https://doi.org/10.3389/fpsyg.2016.00898
53. Vansteenkiste, M., Williams, G. C., \& Resnicow, K. (2012). Toward systematic integration between self-determination theory and motivational interviewing as examples of top-down and bottom-up intervention development: autonomy or volition as a fundamental theoretical principle. International Journal of Behavioral Nutrition and Physical Activity, 9(1), 1-11. https://doi.org/10.1186/1479-5868-9-23

54. Vlachopoulos, S. P., Kaperoni, M., \& Moustaka, F. C. (2011). The relationship of selfdetermination theory variables to exercise identity. Psychology of sport and exercise, 12(3), 265-272.

https://doi.org/10.1016/j.psychsport.2010.11.006

55. Williams, G. C., \& Deci, E. L. (1996). Internalization of biopsychosocial values by medical students: a test of self-determination theory. Journal of personality and social psychology, 70(4), 767-779. https://doi.org/10.1037/0022-3514.70.4.767

56. Williams, G., Freedman, Z., \& Deci, E. (1998). Supporting autonomy to motivate glucose control in patients with diabetes. Diabetes, 47(1S). https://doi.org/10.2337/diacare.21.10.1644

57. Yaneva, G. A., Dimitrova, T. S., Cherneva, D. P., Ivanova, N. D., Fialkovska, N. A., Vachkov, I. H., .. \& Ivanov, D. L. (2018). Quantitative dermatoglyphic study of the finger ridge count in breast carcinoma patients from NortheastĐ $\mu$ rn Bulgaria. Scripta Scientifica Salutis Publicae, 4, 51-55. https://doi.org/10.14748/sssp.v4i0.4335

58. Zhou, Y. (2021). Regressing Dual Side of Motivation on Exercise Commitment in Adults: A Structured Equation Modelling Approach. Revista de Psicología del Deporte, 30(1), 60-74.BayHinitz, A.K., Peterson, R.F., Quilitch, H.R. (1994). Cooperative games: A way to modify aggressive and cooperative behaviors in young children. Journal of Applied Behavior Analysis, 27(3), 435-446. 